\title{
A Correlative Method for Visualizing Fluorescent Fusion Proteins in Plant Cryostat Sections by Confocal and Transmission Electron Microscopy
}

\author{
S. Modla*, J-Y Lee** and K. J. Czymmek* \\ "Delaware Biotechnology Institute, Bio-imaging Center, University of Delaware, 15 Innovation \\ Way, Newark, DE 19711 \\ ** Department of Plant and Soil Sciences, University of Delaware, Newark, DE 19711
}

Correlative light and electron microscopy allows the same targeted biological structure of interest to be analyzed with multiple imaging platforms. Current correlative methods that utilize quantum dots [1], FluoroNanogold ${ }^{\mathrm{TM}}$ [2] and DAB photo-oxidation reactions [3] are highly effective at marking the same structure for light and electron microscopy, but pinpointing rare structure within thickwalled plant tissues remains problematic. Here we present a correlative technique that allows highly specific fluorescent protein localizations within plants to be readily targeted for TEM analysis while maintaining morphological preservation.

Transgenic Arabidopsis seedlings that express a YFP marker were fixed in the dark in 4\% paraformaldehyde $0.1 \%$ glutaraldehyde in $0.2 \mathrm{M}$ Sorensen's phosphate buffer overnight at $4^{\circ} \mathrm{C}$, washed and infiltrated with a gradient of $2.3 \mathrm{M}$ sucrose in buffer. The cryo-protected samples were frozen and 50- $\mu \mathrm{m}$ thick cryo-sections were collected onto adhesive Permanox ${ }^{\circledR}$ microscope slides using the CryoJane ${ }^{\circledR}$ Tape-Transfer System. Sections were mounted in SlowFade Gold and imaged with a Zeiss LSM 5 DUO confocal microscope. Following confocal mapping of cross walls containing plasmodesmata (PD) pit fields, samples were processed for TEM. Briefly, samples were fixed overnight in $2 \%$ glutaraldehyde and $2 \%$ paraformaldehyde in $0.1 \mathrm{M}$ sodium cacodylate buffer $\mathrm{pH} 7.4$, washed, postfixed in $1 \%$ osmium tetroxide, rinsed, dehydrated in a graded ethanol series, and infiltrated and flat-embedded with Embed-812 epoxy resin. Samples were serial sectioned using a Diatome diamond knife, collected onto slotted grids and post-stained with uranyl acetate and Reynolds' lead citrate. Data was acquired with a Zeiss Libra 120 PLUS TEM at $120 \mathrm{kV}$ equipped with a Gatan Ultrascan 1000 2k x 2k CCD camera.

PD pit fields at cross walls between cortex cells were readily identified by the presence of areas containing punctate YFP fluorescence (Figs. 1A-B). Using confocal maps and z-stacks as a guide, selected regions were targeted for TEM (Figs. 1C-E). Serial sections allowed the entire cross wall to be imaged, and corresponding PD were directly correlated to confocal microscope data (Figs. 1B-C, E). Overall, the cells were extremely well preserved, without evidence of ice crystal damage and the YFP marker was confirmed to localize to plasmodesmata. Analyzing rare nano structures by TEM is an arduous task, requiring much time and effort. The technique presented here facilitates identification of subcellular structures tagged with fluorescent markers, while preserving ultrastructural integrity, by first sectioning cryo-protected material into manageable segments, mapping the region of interest by confocal and then imaging the same area by TEM.

References

[1] R. Nisman et al. J. Histochem. Cytochem. 52 (2004). 13.

[2] J. M. Robinson and D. D. Vandré. J. Histochem. Cytochem. 45 (1997). 631.

[3] T. J. Deerinck et al. J. Cell Biol. 126. (1994). 901. 
[4] We would like to acknowledge Dr. Jeff Caplan for assistance with confocal imaging and Megan Kasprzak for her help with image analysis.
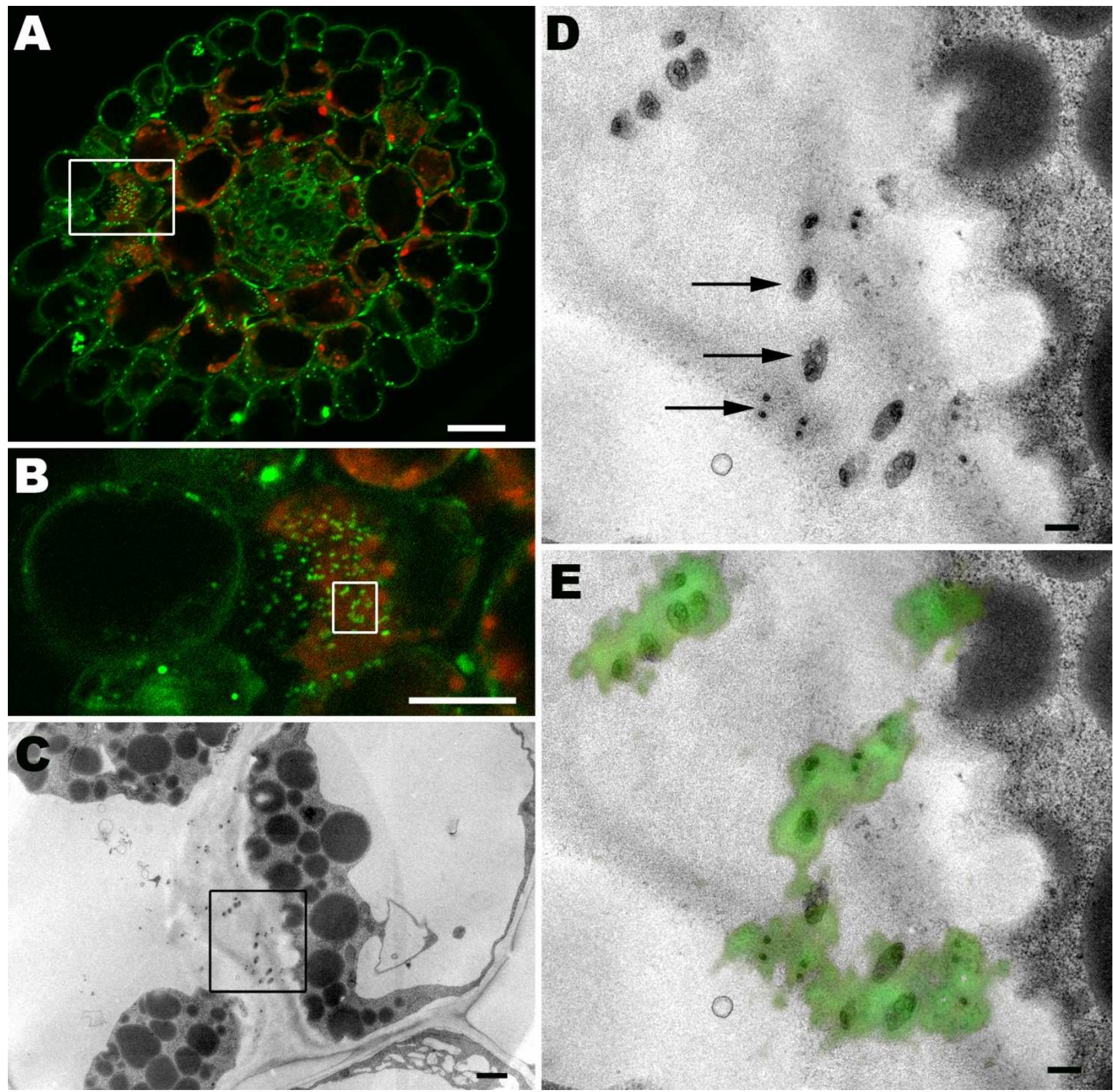

FIG. 1. Confocal and TEM images correlating YFP-labeled plasmodesmata pit fields. A. Confocal image of a cryostat cross-section of a cryo-protected transgenic Arabidopsis hypocotyl. The boxed area contains cross walls that were targeted for TEM. Scale bar $20 \mu \mathrm{m}$. B. Zoomed image from the boxed area in 1A. PD in the boxed area were correlated by TEM. Scale bar is $10 \mu \mathrm{m}$. C. TEM. PD bounded by the box correlate to those in 1B. Scale bar $1 \mu \mathrm{m}$. D. High magnification micrograph of the PD (arrows) depicted in the boxed regions in 1B and C. Scale bar is $0.2 \mu \mathrm{m}$. E. Confocal-TEM overlay showed the localization of YFP fluorescence to the clusters of PD. Scale bar is $0.2 \mu \mathrm{m}$. 